\title{
A child with Morquio syndrome and mixed mitral valve disease
}

\author{
D S G Mettananda ${ }^{1}$ and Aswini D Fernando ${ }^{2}$
}

\section{Introduction}

Mucopolysaccaharidoses (MPSs) are a group of inherited storage diseases caused by a deficiency of lysosomal enzymes that degrade glycosaminoglycans (GAGs). The MPSs are a heterogenous group characterised by intra-lysosomal accumulation of GAGs, excessive urinary excretion of GAGs, and variable degrees of progressive mental and physical deterioration [1]. Mucopolysaccharidosis type IV (Morquio syndrome) is autosomal recessively inherited. The primary pathology is defective degradation of keratan sulphate due to one of two enzyme deficiencies; n-acetylgalactoseamine-6-sulfate (chromosome 16q) or $\beta$-galactosidase (chromosome $3 \mathrm{p}$ ) [1,2].

\section{Case report}

A 10-year old child was admitted to hospital with a history of fever, cough and exertional dyspnoea for 5 days. She was clinically diagnosed to have Morquio syndrome at the age of 2 years and had short stature, skeletal dysplasia, corneal clouding and normal intelligence. She gave a history of frequent episodes of shortness of breath during the past 2 years.

On examination her height and weight were both well below the third centile. She had a short trunk, pectus carinatum, corneal clouding, and short stubby hands and fingers. She was tachypnoeic, and using accessory muscles of respiration. On auscultation bilateral basal crackles were heard. She had also tachycardia and cardiomegaly. There was a pansystolic murmur and a diastolic murmur best heard at the apex. Liver was palpable $3 \mathrm{~cm}$ below the right costal margin and the spleen was just palpable. Neurological examination was normal except for a waddling gait.

Chest xray showed cardiomegaly, double and straight left heart border and plethoric lung fields (figure). ECG showed bifid $\mathrm{P}$ waves (P mitrale). 2-D echocardiogram showed a dilated left atrium, moderate mitral stenosis, grade III mitral regurgitation and mild tricuspid regurgitation.

\section{Discussion}

Characteristic features of Morquio syndrome are short-trunk dwarfism, skeletal dysplasia (including thoraco-lumbar kyphosis, atlanto-axial subluxation, anterior vertebral beaking, pectus carinatum, rib abnormalities, coxa valga, genu valgum, epiphysial/ metaphysical irregularities and small irregular metacarpals, corneal clouding, and waddling gait with preservation of intelligence $[1,2]$.

Cardiac involvement is well recognised in most forms of mucopolysaccharidoses but there is poor documentation of abnormalities specific to Morquio syndrome. Cardiac involvement is usually of regurgitant mitral and aortic valve lesions due to cardiomyopathy [2]. In a study of 10 patients of Morquio syndrome, 5 patients had mitral valve involvement and 4 had aortic valve involvement. Only a single patient had mitral valve thickening to the point of mitral stenosis. All cardiac lesions were mild and haemodynamically stable [3]. A search of the literature revealed only this one reported case of mitral stenosis in a child with Morquio syndrome.

The index patient had clinical, radiological, and echocardiographical evidence of moderate mitral stenosis and moderate to severe haemodynamic instability. This is the second reported case of mitral stenosis in a child with Morquio syndrome and in contrast to the previous case, this child has severe mitral valve disease with haemodynamic instability. She was started on medical treatment with diuretics and calcium channel blockers and is awaiting mitral valve replacement.

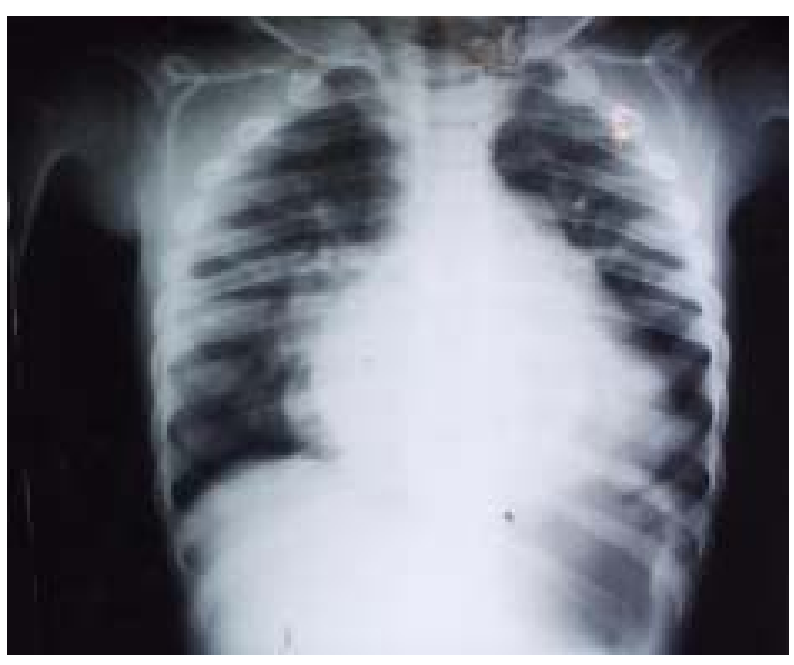

Figure. Chest xray showing cardiomegaly, straight left heart border and plethoric lung fields

${ }^{1}$ Lecturer, and ${ }^{2}$ Senior Lecturer, Department of Paediatrics, Faculty of Medicine, University of Kelaniya, Sri Lanka, Correspondence: ADF, e-mail <aswinif@sltnet.lk>. Received 24 October, and accepted 21 November 2007. Conflicts of interest: none declared. 


\section{References}

1. Berham RE, Kliegman RM, Jenson HB, eds. Nelson Textbook of Pediatrics. 17th ed. Philadelphia: Saunders, 2004: 482-6.
2. Lankester BJA, Whitehouse M, Gargan MF. Morquio syndrome. Current Orthopaedics 2006; 20: 128-31.

3. John RM, Hunter D, Swanton RH. Echocardiographic abnormalities in type IV mucopolysaccharidosis. Archives of Diseases in Childhood 1990; 65: 746-9. 